\title{
DESINTERÉS DE LOS ESTUDIANTES DE BÁSICA SUPERIOR EN EL PROCESO DE EVALUACIÓN FORMATIVA DE LOS APRENDIZAJES
}

Lic. Gladys Adriana Valencia Coca ${ }^{1}$ M.Sc. Velasteguí López Luis Efraín ${ }^{2}$

\begin{abstract}
Education in Ecuador is the cornerstone in the development of society, which helps to improve the quality of life of families and therefore of the country, but because of the great demands that arise in today's globalized world, institutions basic education are aimed towards a comprehensive education in the teaching process to students, to promote their skills and potential, based on values, from this perspective is taken into account the evaluation process that determines how knowledge acquired systemically during educational process taking into account planning and skills achieved. The learning evaluation process considers the ethical commitment of teachers to develop and use techniques and suitable to measure knowledge acquired by pre-planning student assessment instruments tools, based on criteria of relevance, materiality and efficiency, as required in the social and cultural context, we aimed to measure the socio-emotional and intellectual abilities of students; with the intention of revealing the evaluation standards for students to feel interest in the evaluation process, the present study, culminating in the criteria for the development of instruments for educational learning assessment of students in Basic Education High School Ernesto Ophuls.
\end{abstract}

Keywords: Evaluation Methodology, Strategies, Evaluation Standards, Evaluation Techniques, Educational Assessment.

CÓDIGO UNESCO: Educación 531204

\section{RESUMEN}

La educación en el Ecuador es el pilar fundamental en el desarrollo de la sociedad, que ayuda a mejorar la calidad de vida de las familias y por ende del país, pero

\footnotetext{
${ }^{1}$ Unidad Educativa del Milenio, Napo,Ecuador, adrianavc_0586@yahoo.es

${ }^{2}$ Universidad Técnica de Ambato, Ambato, Ecuador le.velastegui@uta.edu.ec
} 
debido a las grandes exigencias que se presentan en el mundo actual globalizado, las instituciones de educación básica están encaminadas hacia una formación integral en el proceso de enseñanza a los estudiantes, que promueva sus habilidades y potencialidades, basadas en valores, desde esta perspectiva se toma en cuenta el proceso de evaluación que determina cuantos conocimientos adquieren de forma sistémica durante el proceso educativo tomando en cuenta la planificación y las destrezas alcanzadas. El proceso de evaluación del aprendizaje considera el compromiso ético de los docentes, para que desarrollen y utilicen técnicas y herramientas adecuadas para medir el conocimiento que adquieren los estudiantes previo a la planificación de los instrumentos de evaluación, en base a criterios de pertinencia, relevancia y eficiencia, según las necesidades en el contexto social y cultural, encaminado a medir las capacidades intelectuales y socio-afectivas de los estudiantes; con la intención de develar los estándares de evaluación para que los estudiantes sientan interés en el proceso de evaluación, el presente estudio, culmina con los criterios para el desarrollo de los instrumentos de evaluación educativa del aprendizaje de los estudiantes de Educación Básica Superior de la Escuela Ernesto Ophuls.

Palabras clave: metodología de evaluación, estrategias, estándares de evaluación, técnicas de evaluación, evaluación

\section{INTRODUCCIÓN}

En América Latina las principales transformaciones en la educación siempre están vinculadas con cambios en los sistemas de evaluación. A menudo esto ocurre en momentos importantes de definición de nuevos rumbos en la sociedad, ya que en las instituciones educativas aparecen soluciones para problemas antiguos y nuevos que los políticos y las élites económicas no logran resolver.

La educación se está constituyendo en un instrumento de la economía. Y la evaluación en la educación tendrá que adaptarse a la función económica, con conceptos relacionados con la productividad, excelencia, gestión racional, etc., convirtiéndose en el resultado de criterios 
y procedimientos de dimensión cuantitativa y nociones de eficiencia en el aprendizaje de los estudiantes, basada en el interés de encontrar recursos y procedimientos que de una manera objetiva y confiable que reflejen el aprendizaje, y ayude a los estudiantes a saber cómo y en que deben mejorar su propio rendimiento.

En el contexto ecuatoriano, en muchos de los casos las práctica de la evaluación tradicionales han permanecido inalterables, se hace necesario impulsar un cambio, fomentando una evaluación que eduque, es decir que sea formativa y que permita a los estudiantes, en su diversidad, conocer y ser capaces de desarrollar sus potencialidades, hasta llegar a ser individuos autorregulados, capaces de liderar su propio aprendizaje y estableciendo políticas fundamentales educativas basadas en la experiencia que impulsen un mayor desarrollo en los estudiantes tanto a nivel académico como motivacional y por ende personal.

En la Escuela de Educación Básica Ernesto Ophuls, la necesidad de promover un aprendizaje auténtico y significativo, vinculada a la evaluación debe, además de medir, educar y permitir valorar el desempeño de estudiante para en base a esta información tomar decisiones que permitan optimizar el proceso de enseñanza - aprendizaje.

La institución se basas en el marco legal, de la evaluación, calificación y promoción de los estudiantes (LOEI), por lo que la Institución cuenta con los parámetros basados en la evaluación con la obtención de resultados, lo que hace que los estudiantes al tener una mayor cantidad de oportunidades, no realicen el esfuerzo necesario por aprovechar las primeras evaluaciones, y se confían en que tienen un mayor número de oportunidades que a la larga o corta les va ayudar para ser promovidos al siguiente año lectivo, creando desinterés en el proceso de evaluación.

Luego del análisis del problema a investigarse sobre el desinterés en el proceso evaluativo de aprendizaje de los estudiantes de Básica Superior en la Escuela Ernesto Ophuls, se puede describir que una de las causas es la elaboración de inadecuados instrumentos de evaluación, lo que hace que los estudiantes tengan un bajo rendimiento académico, ya que 
al diseñar un instrumento que no aporte adecuadamente a la evaluación de las destrezas no se estaría promoviendo al aprendizaje integral del estudiante.

Las varias oportunidades en el proceso de evaluación contemplada en la LOEI, existe una inadecuada potencialización en las destrezas que se espera que los estudiantes las tengan al culminar el año lectivo, provocando el desinterés y que no se esfuercen en el proceso de evaluación del aprendizaje.

Además la falta de apoyo y control de los padres de familia en el proceso de aprendizaje, hace que los estudiantes se encuentren desmotivados, ya que por motivos de horarios de trabajo o porque los padres no han culminado sus estudios primarios, no les pueden ayudar a desarrollar sus tareas y deberes o simplemente económicamente no cuentan con los materiales necesarios.

Teniendo como objetivos analizar el desinterés de los estudiantes de Básica Superior en el proceso de evaluación formativa en la Escuela Ernesto Ophuls del Cantón Tena Provincia de Napo, mediante la descripción de las etapas de evaluación formativa que se aplica a los estudiantes en el proceso de enseñanza-aprendizaje, determinando el desinterés en el proceso evaluativo de los estudiantes de Básica Superior de la Escuela Ernesto Ophuls, para finalizar diseñando una guía de procedimientos de las etapas de la evaluación formativa en el desempeño académico de los estudiantes para la Escuela Ernesto Ophuls del Cantón Tena, Provincia de Napo.

Todo sustentado en el paradigma socio propositivo con enfoque cuanti-cualitativo, cuantitativo porque los resultados de la investigación (test, encuestas, entrevistas) serán sometidos a análisis numéricos con el apoyo de la estadística, y cualitativo porque estos resultados numéricos serán interpretados críticamente con el apoyo del marco teórico.

En el desarrollo de la investigación se plantea la modalidad bibliográfica, que tiene como propósito conocer, comparar, profundizar y deducir los enfoques, teorías y conceptualizaciones de diversos autores, en esta investigación se sustentará en la recopilación de información en libros, tesis o proyectos, folletos, artículos científicos a través del internet; acorde a los enfoques y teorías de las variables de estudio, es decir la manera de recolección de información será de forma directa.

Es por eso que se plantea desarrollar el estudio sobre el desinterés de los estudiantes de Básica Superior en el proceso de evaluación formativa de los aprendizajes en la Escuela 
Ernesto Ophlus, para mejorar los procesos y conseguir un mayor desarrollo en las capacidades y potencialidades de los estudiantes.

\section{DESARROLLO}

En la Escuela Ernesto Ophuls durante el proceso de enseñanza - aprendizaje se presentan problemas en el proceso de evaluación debido al desinterés que tienen los estudiantes, teniendo estudiantes poco preparados y con la facilidad de aplicar las destrezas que se debe tener al finalizar los años escolares.

Se ha determina que en el proceso de enseñanza - aprendizaje una parte importante es la evaluación, es así que se realiza una investigación sobre el proceso de evaluación y el desinterés de los estudiantes.

Dada la multiplicidad de variables que inciden en las prácticas docentes y de evaluación, las profundas raíces de algunas de ellas, y el hecho de que modificar otras no esté al alcance de los maestros mismos, sino que involucra a otras instancias del sistema educativo y a los padres de familia, las actividades de actualización que tengan como propósito transformar en profundidad las prácticas de evaluación de los maestros no podrán consistir únicamente en talleres breves, esporádicos y superficiales. La conclusión de Schneider y Randel, es que:

...los esfuerzos de actualización sobre evaluación formativa en aula deben tener una duración sostenida con suficientes horas presenciales para presentar los conceptos y ofrecer apoyo sustancial que permita poner en práctica la aplicación de nuevas habilidades. La duración óptima de los programas de actualización está por determinarse. Supovitz y Turner han encontrado que los maestros necesitan entre 40 y 80 horas de actualización para que sus prácticas cambien respecto a las del promedio... los maestros que trabajan en escuelas de bajos resultados pueden modificar su base de conocimientos sobre evaluación formativa en aula en 30 a 40 horas; sin embargo, el número de horas necesario para cambiar los conocimientos de los maestros no es necesariamente el mismo que bastará para que el rendimiento de los alumnos aumente (Schneider y Randel, 2010: 272-273). 
La última idea es fundamental: es necesario modificar los conocimientos del maestro sobre evaluación para que sus prácticas cambien, pero esto no basta; tampoco será suficiente llevar a cabo talleres de 40-80 horas de duración. Es indispensable un esfuerzo continuado durante uno o dos ciclos escolares, basado en el trabajo conjunto de una comunidad de aprendizaje formada por los maestros de una o varias escuelas. En este sentido, Black y Wiliam dicen:

Los maestros necesitan una variedad de ejemplos vivos para la implementación de tales prácticas por parte de colegas con los que puedan identificarse y de los que puedan derivar la convicción y la confianza de que ellos también pueden hacerlo mejor, y constatar qué quiere decir en la práctica "hacerlo mejor” (2004: 21).

Si bien los maestros aprecian las recomendaciones prácticas, las acciones de evaluación formativa se pueden volver mecánicas y rituales si no se promueve la reflexión sobre los principios que las sustentan. Los valores, las creencias y las prácticas no son uniformes entre los maestros; aunque la mayoría tiene valores educativos claros y positivos, la mayoría encontró dificultad para alinear las nuevas prácticas y sus valores.

La influencia más importante resultó ser la indagación colaborativa entre los maestros sobre sus prácticas... que se puede extender más allá del aula por medio de redes dentro de la escuela y entre escuelas. Lo anterior depende en buena medida de las estructuras organizacionales, la cultura y el liderazgo. El reto clave para los directivos es, pues, crear el espacio y el clima para que el personal de las escuelas pueda reflexionar sobre su práctica y compartir esa reflexión (James, 2010: 169-170).

Para que puedan extenderse las nuevas prácticas será necesario, pues, que las instancias de dirección y supervisión ofrezcan un apoyo consistente, además de que estén presentes condiciones de trabajo y recursos de la escuela y el aula mínimamente suficientes, y que se remuevan los obstáculos que presentan una normatividad inadecuada y evaluaciones externas de enfoque incompatible con el de evaluación formativa bien entendido. Así mismo, habrá que contar con el apoyo de unos padres de familia enterados de la importancia y el sentido de los cambios. Conviene subrayar tres puntos: 
La práctica de adecuadas evaluaciones acorde al medio en el que se desarrollan los estudiantes y con un enfoque formativo es incompatible con un currículo demasiado extenso, sobrecargado de contenidos, que obliga a los maestros a dedicar poco tiempo a cada tema y les impide actuar sobre los complejos procesos que implica modificar las concepciones de los alumnos, y con el sistema educativo que más tiempo los docentes deben pasar horas planificando que aplicando esas mismas planificaciones hace que el proceso de evaluación no se pueda realizar en su totalidad y enfocados a lo que verdaderamente se necesita desarrollar en los estudiantes.

Dentro de las Estrategias de evaluación de los aprendizajes centrados en el proceso, se procura destacar el valor intrínseco de la evaluación como motor de cambio y mejora. Cuando el acento se coloca en el proceso de evaluación y se imbrica con el proceso de aprendizaje, la evaluación adquiere un potencial formativo y de "empowerment" que va mucho más allá en la formación de la persona que en el mero hecho de constatar avances u objetivos conseguidos. Desde esta perspectiva el énfasis se proyecta en el proceso más que en el resultado introduciéndose, en lo que se está haciendo, la reflexión pedagógica sobre lo que se hace, cómo se hace y que utilidad tiene. La evaluación cuando se realizada de esta manera, desde una visión innovadora y crítica, no hay duda que incide de forma notable en la calidad de los procesos de aprendizaje de contenidos y formación de la persona. (Encinas M, 2010:26)

Según Encinas M, (2010), se formula un conjunto de enunciados que orienta las prácticas evaluativas:

- Evaluar y aprender son dos procesos que se autoalimentan.

- La visión de la evaluación como proceso para aprender es más prometedora que como valoración de resultados conseguidos.

- La evaluación debe traspasar la frontera de los objetivos y estar abierta a lo no planeado, incierto, imprevisto e indeterminad.

- Las estrategias de evaluación cualitativa que ponen en evidencia el proceso de aprendizaje que se realiza y no meramente sus resultados favorecen aprendizajes profundos. 
- Las estrategias que se utilicen en la evaluación contribuyen al aprendizaje de la evaluación.

Aprender a evaluar evaluando es una afirmación que ha de estar presente en las aulas a fin de que los estudiantes sean personas capaces de dirigir con responsabilidad sus procesos de aprendizaje en todos los órdenes de la vida.

Proyecto principal de educación en América Latina y el Caribe, La experiencia de América Latina, Robin Horn, Laurence Wolff y Eduardo Vélez (2012), se tienen las siguientes conclusiones:

- Chile ha sido el país que más éxito ha tenido en las evaluaciones iniciadas en 1978. Después de una serie de problemas iniciales, incluyendo la oposición de profesores y alumnos, el programa establecido en 1988 ha logrado medir exitosamente el aprendizaje alcanzado por universos de alumnos de cuarto y octavo años de educación básica. El relativo éxito del programa chileno parece ser el resultado de la alta calidad del personal y un fuerte énfasis en proporcionar retroinformación directa a los profesores, colegios y distritos, así como en informar al público general sobre la naturaleza y papel del programa de evaluación.

- Costa Rica comenzó su programa en 1986 y 1987 evaluando el universo de alumnos de tercero, sexto y noveno años de educación primaria. El programa no especificó claramente su objetivo, pero sus metas implícitas incluyeron el uso de las evaluaciones como herramienta para debatir públicamente la necesidad de mayor financiamiento para la educación primaria, y convencer al público acerca de la necesidad de volver a introducir el uso de pruebas parcialmente estandarizadas como requisito para que Sistemas de evaluación educacional en alumnos egresados de educación secundaria pudieran obtener certificado de estudios.

- El gobierno de México ha efectuado una gran cantidad de diagnósticos y evaluaciones durante un período de veinte años. También ha hecho un análisis del examen de ingreso a educación secundaria, con fines de evaluación. El organismo encargado de la mayor parte de esta tarea ha perdido, recientemente, una considerable cantidad de buen 
personal además de contar con un presupuesto insuficiente. Los resultados han sido diseminados, pero en forma excesivamente teórica y general.

- Para Colombia, el desafío consiste en aumentar esta capacidad mediante la utilización de los exámenes de admisión para fines evaluativos, similares a los de Kenia, así como de desarrollar un sistema de evaluación para la educación primaria. Ninguno de los cuatro países ha incorporado adecuadamente la investigación a las evaluaciones y pruebas. Esto implica que gran parte del valor del esfuerzo desplegado se está perdiendo debido a la falta de elementos complementarios adicionales. Además, frecuentemente hay una tendencia a usar el universo de alumnos en lugar de muestras menos onerosas. El uso de métodos de muestreo depende de gran pericia estadística, la cual es un bien muy escaso.

Por otra parte, cabe mencionar que ninguno de los cuatro países ha hecho el esfuerzo necesario en la diseminación y seguimiento de los resultados obtenidos en las pruebas. Chile tiene hasta el momento el mejor desempeño en este sentido, pero aún queda mucho por hacer para lograr un buen impacto en el comportamiento dentro de la sala de clases. Los esfuerzos de diseminación realizados en México, a pesar de su extensión, han sido excesivamente generales y teóricos y tampoco se ha entregado sistemáticamente la información a nivel de colegios o distritos individuales. Los esfuerzos de diseminación efectuados en Costa Rica fueron hechos en forma ad hoc por una organización ajena al gobierno. (Horn, R. Wolff, L y col, 2012)

Esta investigación tiene su sustento legal en la Constitución de la República del Ecuador, la misma que responde al cumplimiento de las normas jurídicas, legales, organismos oficiales de la inspección, control y vigilancia de la educación y de carácter institucional.

Basados en la Ley Orgánica De Educación Intercultural, se va a tomar en cuenta los siguientes artículos que competen al proceso de evaluación:

Art. 186.- Tipos de evaluación. La evaluación estudiantil puede ser de los siguientes tipos, según su propósito: 
1. Diagnóstica: Se aplica al inicio de un período académico (grado, curso, quimestre o unidad de trabajo) para determinar las condiciones previas con que el estudiante ingresa al proceso de aprendizaje;

2. Formativa: Se realiza durante el proceso de aprendizaje para permitirle al docente realizar ajustes en la metodología de enseñanza, y mantener informados a los actores del proceso educativo sobre los resultados parciales logrados y el avance en el desarrollo integral del estudiante; $y$,

3. Sumativa: Se realiza para asignar una evaluación totalizadora que refleje la proporción de logros de aprendizaje alcanzados en un grado, curso, quimestre o unidad de trabajo.

Art. 196.- Requisitos para la promoción. La calificación mínima requerida para la promoción, en cualquier establecimiento educativo del país, es de siete sobre diez (7/10).

En los subniveles de Básica Elemental y Básica Media, para la promoción al siguiente grado, se requiere una calificación promedio de siete sobre diez (7/10) en cada una de las siguientes asignaturas: Matemática, Lengua y Literatura, Ciencias Naturales y Estudios Sociales, y lograr un promedio general de todas las asignaturas de siete sobre diez (7/10). DEROGADO.27 de junio de 2014.

\section{Reformas al Reglamento General a la Ley Orgánica de Educación Intercultural}

Artículo 10.- Sustitúyase el segundo inciso del artículo 196, por el siguiente:

"En los subniveles de Básica Elemental y Básica Media, para la promoción al siguiente grado se requiere una calificación promedio de siete sobre diez (7/10) en el conjunto de las asignaturas que componen la malla curricular."

En el subnivel de Básica Superior y el nivel de Bachillerato, para la promoción al siguiente grado o curso, se requiere una calificación promedio de siete sobre diez (7/10) en cada una de las asignaturas del currículo nacional.

Las asignaturas adicionales al currículo nacional que cada establecimiento definiere en su Proyecto Educativo Institucional, correspondientes a la innovación curricular que estuviere debidamente aprobada por el Nivel Zonal respectivo, serán requisitos para la promoción 
dentro del establecimiento; sin embargo, no lo serán si el estudiante continúa sus estudios en otra institución educativa.

Además para la presente investigación se tomará las bases legales del Código de la Niñez y la Adolescencia, que enfoca a los derechos de los estudiantes.

Art.37. Derecho a la Educación. Los niños/as y adolescentes tienen derecho a una educación de calidad, este derecho demanda de un sistema educativo que Garantice el acceso y permanencia de todo niño/a a la Educación Básica. Todos los niños/as sin distinción, ni excepción recibirán una educación de calidad garantizando el desarrollo permanente del niño/a.

La investigación basándose en la Ley Orgánica de Educación Intercultural y el Código de la Niñez y Adolescencia tiene como objetivo primordial, velar por los derechos de los estudiantes y hacer de la investigación un proceso que ayude al mejoramiento de la calidad educativa en beneficio de los estudiantes de Básica Superior de la Escuela "Ernesto Ophuls".

\section{CONCLUSIONES.}

- Descripción de las etapas de evaluación que se aplica a los estudiantes dentro del proceso de aprendizaje, realizar reajustes acordes a los estándares de calidad tomando en cuenta el contexto social y cultural de la Escuela, para la mejora en los procesos de evaluación formativa, respondiendo de manera satisfactoria a los requerimientos de la sociedad actual.

- El presente proyecto pretende determinar las causas del desinterés de los estudiantes en el proceso de evaluación formativa, tomando en cuenta el proceso de evaluación, la planificación, las técnicas e instrumentos de evaluación acorde a las necesidades de los educandos, que les permita alcanzar las destrezas necesarias para alcanzar el éxito tanto académico.

- Diseño de una guía de procedimientos de las etapas de evaluación formativa en el desempeño académico de los estudiantes de la Escuela que mida de una forma 
confiable los conocimientos adquiridos que potencien sus habilidades $\mathrm{y}$ capacidades.

RECEIVED: JANUARY, 2017

REVISED: MARCH, 2017

\section{Referencias Bibliográficas}

- Álvaro Andrés Rivera Sepúlveda, Diana Carolina Jaimes Suárez, César Augusto Pulga Cruz, 2012, Fundamentación epistemológica de una investigación en educación, $\begin{array}{lllll}\text { Revista Ciela Universidad de la } & 23\end{array}$ http://revistas.lasalle.edu.co/index.php/ls/article/view/767/683

- Bordas M, Cabrera F, 2010, ESTRATEGIAS DE EVALUACIÓN DE LOS APRENDIZAJES CENTRADOS EN EL PROCESO Universidad de Barcelona, Revista Española de Pedagogía. Año LIX, enero-abril, n.218.pp.25 a 48 http://cmapspublic3.ihmc.us/rid=1GLSW84JS-WYZWX0-

H40/Evaluaci\%C3\%83\%C6\%92\%C3\%82\%C2\%B3n\%20del\%20Proceso $\% 20 \mathrm{de} \% 20 \mathrm{~A}$ prendizaje.pdf

- Encinas M, 2010, Alineamiento constructivo de la enseñanza y su relación con la calidad del aprendizaje en estudiantes de la Escuela de Educación Secundaria, Facultad de Educación, UNAP, Iquitos-2009-II, Lima-Perú. http://cybertesis.unmsm.edu.pe/bitstream/cybertesis/1696/1/encinas_pm.pdf

- Felipe Martínez Rizo, 2012, Dificultades para implementar la evaluación formativa Vol. XXXV, núm. $\quad$ 139, 2013|IISUE-UNAM http://www.scielo.org.mx/pdf/peredu/v35n139/v35n139a9.pdf

- Gallegos Pablo, 2005, "Elementos teóricos y metodológicos para la investigación educativa", Unidad 164 de la Universidad Pedagógica Nacional, Zitácuaro, Michoacán, México, pp. 45.

- Martínez, C, 2008, Procesos Evaluativos en la Práctica Pedagógica de los Maestros en Formación. Institución Educativa Normal San Carlos -La Unión Nariño, San Juan de Pasto.

http://repository.lasalle.edu.co/bitstream/handle/10185/1668/T75.08\%20A48p.pdf?sequ ence $=1 \&$ isAllowed $=\mathrm{y}$ 
- OCDE, 2004, Evaluación formativa: mejora del aprendizaje en las aulas de secundaria, ISBN-92-64-00739-3 C OECD 2004 http:/www.oecd.org/edu/ceri/34313907.pdf

- Pérez Ma., Castellanos M, Díaz M., González J, Núñez J, 2013, Dificultades de aprendizaje en estudiantes universitarios de primer año ISSN 0716-1840 http://www.scielo.cl/pdf/atenea/n508/art_10.pdf

- Pedro Ravela, Uruguay; Patricia Arregui, Perú; Gilbert Valverde, Estados Unidos; Richard Wolfe, Canadá; Guillermo Ferrer, Argentina; Felipe Martínez Rizo, México; Mariana Aylwin, Chile; Laurence Wolff, Estados Unidos, 2008, Las evaluaciones educativas que América natina necesita http://200.6.99.248/ bru487cl/files/EVals_AL.pdf

- Robin Horn, Laurence Wolff y Eduardo Vélez, 1992, Sistemas de evaluación educacional en América Latina, Boletín PROYECTO PRINCIPAL DE EDUCACION en América Latina y el Caribe, BOLETIN 27, Santiago, Chile, Abril 1992 http://unesdoc.unesco.org/images/0009/000919/091968s.pdf

- Soto, R. E. P. (2011). EXPERIENCIA EDUCATIVA EN ARTE VISUAL DISEÑADA BAJO UN MODELO DE AUTORREGULACIÓN DEL APRENDIZAJE CON ESTUDIANTES UNIVERSITARIOS. Revista Mexicana De Investigación Educativa, 16(49), 597-624. Retrieved from http://search.proquest.com/docview/882073709?accountid=36765

- VALLE Antonio, Ramon Gabanach, Julio Gonzales, Jose nuñez, Susana Rodríguez, Pedro Rosário, 2009, PERFILES MOTIVACIONALES EN ESTUDIANTES DESECUNDARIA: ANÁLISIS DIFERENCIAL EN ESTRATEGIAS COGNITIVAS, ESTRATEGIAS DE AUTORREGULACIÓN Y RENDIMIENTO ACADÉMICO, Revista Mexicana de Psicología, Enero 2009; Volumen 26, Número 1, 113-124 http://repositorium.sdum.uminho.pt/Revista_20Mexicana-20texto_20final_202009.pdf

- López M, Buscá F, Foguet C, y Col., 2009, Evaluación formativa y compartida en educación superior. Propuestas, técnicas, instrumentos y experiencias. Editorial: Narcea. Número de páginas: 270 ISBN: 978-84-277-1596-7 http://search.proquest.com/prisma/docview/748347431/fulltextPDF/E0B9654953E7474 $5 \mathrm{PQ} / 1$ ?accountid $=36765$ 
- Código de la niñez y la adolescencia, publicado por la Ley $\mathrm{N}^{\mathrm{o}} 100$, en Registro oficial 737 del 3 de enero del 2013. http://www.registrocivil.gob.ec/wpcontent/uploads/downloads/2014/01/este-es-06-C\%C3\%93DIGO-DE-LA-

NI\%C3\%91EZ-Y-ADOLESCENCIA-Leyes-conexas.pdf

- Ley Orgánica de Educación Intercultural, Decreto No. 1241, http://educacion.gob.ec/wp-content/uploads/downloads/2012/08/LOEI.pdf

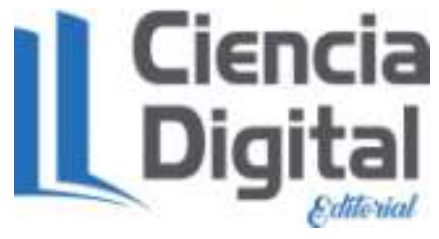

El artículo que se publica es de exclusiva responsabilidad de los autores y no necesariamente reflejan el pensamiento de la Revista Ciencia Digital.

El articulo queda en propiedad de la revista y, por tanto, su publicación parcial y/o total en otro medio tiene que ser autorizado por el director de la Revista Ciencia
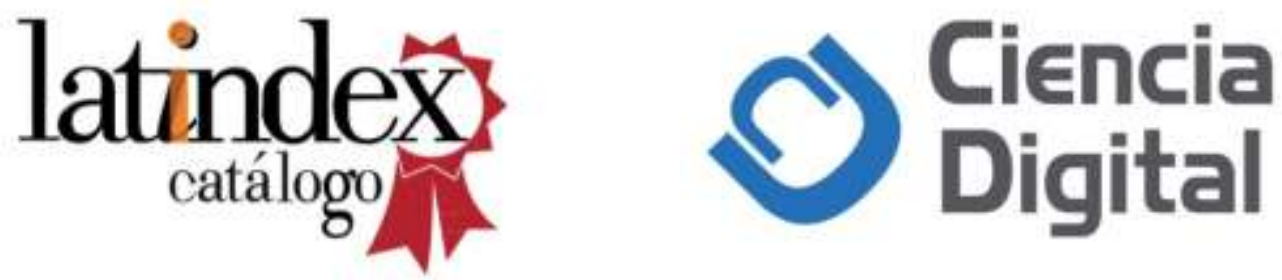